\title{
Is There a Relationship Between Neutrophil/Lymphocyte Ratio and Vitamin D Levels in Individuals Over 65 Years of Age?
}

Merve Yılmaz' ${ }^{1}$ Arzu Gedik², Tevfik Demir ${ }^{3}$, Ayten Eraydın, Dilek Çımrın Abdurrahman Çömlekçi ${ }^{3}$, Sevinç Eraslan ${ }^{3}$

${ }^{1}$ Samsun Gazi StateHospital,EndocrinologyDepartment, Samsun, Turkey

${ }^{2}$ Ağrı StateHospital, EndocrinologyDepartment, Ağrı, Turkey

${ }^{3}$ Dokuz EylulUniversityMedicalFaculty, EndocrinologyDepartment, Izmir, Turkey

${ }^{4}$ GaziantepUniversity MedicalFaculty, EndocrinologyDepartment, Gaziantep, Turkey

${ }^{5}$ Dokuz EylulUniversityMedicalFaculty, BiochemistryDepartment, Izmir, Turkey

\section{OBJECTIVES}

Vitamin Ddeficiency may be an important predisposing factor for infections as well as autoimmune diseases in old individuals. Neutrophil/Lymphocyte Ratio (NLR), is a meaningful indicator of inflammation throughout the body. In this study we aimed to show, whether or not a relationship exists between vitamin $D$ levels and NLR in patients older than 65 years.

\section{METHODS}

Data of 82 patients over 65 years of age was retrospectively examined. Those without chronic renal or liver failure, active infection or disease affecting hemogram values and bone metabolism were included to study.Complete blood count and vitamin D levels of patients were recorded. NLRwas calculated. The correlation analysis was done on patients, in order to understand whether the relationship between vitamin D levels and NLR exists.

\section{RESULTS}

Themeanlevel of vitamin Dwas $21.20 \pm 10.99$ and NLR was2.09 \pm 0.97 . Patientsweredividedinto 4 groupsaccordingtothelevel of vitamin D. Therewasn'tstatisticallysignificantdifferencebetweenthegroups interms of NLR $(p=0.433)$. Whenthepatientsweredividedintotwogroupsaccordingtotheadequate $(>30 \mathrm{ng} / \mathrm{ml})$ andinadequate $(\leq 30 \mathrm{ng} / \mathrm{ml})$ levels of vitamin $D$, therewasn'tsignificantdifferencebetweenthegroups in terms of NLR $(p=0.169)$.

Significantcorrelationwasn'tdetectedbetweenthemean vitamin D levelsand NLR in patients.

Therewasn'tsignificantcorrelationbetween vitamin D levelsand NLR, evenwhendividedinto 3 groupsaccordingtotheir age.

\section{CONCLUSIONS}

An importantrelationshipbetween vitamin D levelsandimmunesystemfunction has beenreported. Increase in NLR, which is an indicator of acutephaseresponse, is associatedwithpoorprognosis in variousdiseases. Inourstudy, therewasn'tsignificantrelationshipbetween vitamin D levelsand NLR. However, thereisn'tanystudydone on thistopic in theliterature. Further studies with higher number of patients may be beneficial. 